Chimia 45 (1991) 319-321

(c) Schweiz. Chemiker-Verband; ISSN $0009-4293$

\section{Supramolekulare Strukturen zur Lichtabsorption}

\author{
Fritz Vögtle*, Martin Bauer, Carlo Thilgen und Peter Knops
}

Abstract. After a definition of and an introduction into supramolecular structures strategies are developed which lead to new material properties. The specific research topics discussed in this context and with this aim include chromoionophores, molecular switches, large cavities embraced by one or more azobenzene units and macrocyclic polyenes. It is demonstrated how light absorption can be manipulated by structural variations of the host structures and of the corresponding supramolecular complexes. The discussion is extended to new dyestuffs bearing small and large cavities, the light absorption of which can be manipulated and even switched selectively by matching metal ions and organic particles (host/guest interaction).

\section{Supramolekulare Strukturen}

Supramolekular nennt man Strukturen, die aus mehreren Partikeln zusammengesetzt sind (s. Fig. I), also Molekülaggregate, die sich - durch nichtkovalente Wechselwirkungen - selbst organisieren. Oft spricht man auch von supramolekularen Wirt/GastKomplexen («molekulare Erkennung»).
Massgeschneiderte Moleküle des Typs II, in denen mehrere verschiedene Untereinheiten miteinander in Wechselwirkung stehen, können - allerdings im weitesten Sinne - gleichfalls als supramolekular angesprochen werden. So können z.B. Wechselwirkungen zwischen den Molekülteilen A und C in II auftreten, wobei der mittlere Molekülteil B (der etwa aus einer Azobenzol-
Fig. 1. Beispiele supramolekularer Strukturen (schematisch)

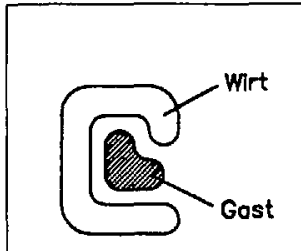

I

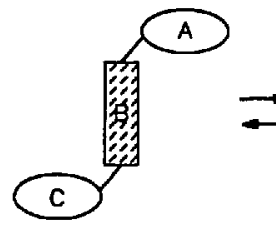

II

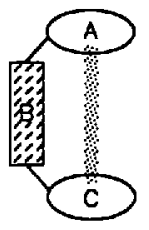

\section{II}

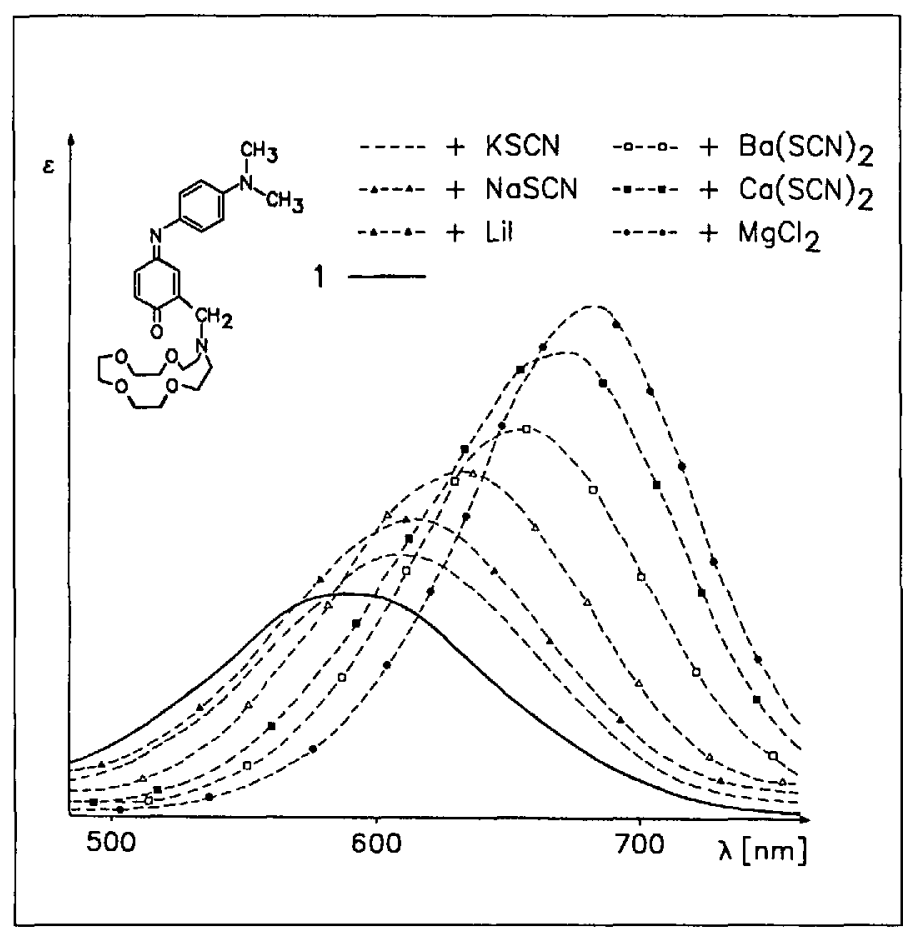

Gruppierung bestehen kann) z.B. durch Lichteinwirkung beeinflusst werden. Das Supramolekül II kann daher als molekularer Schalter fungieren, wenn die Azo-Gruppe (B in II) mit Licht von der $(E)$ - in die (Z)Konfiguration übergeführt und damit die Wechselwirkung zwischen $\mathrm{A}$ und $\mathrm{C}$ beeinflusst («getriggert») wird.

\section{Chromoionophore}

Bei den Chromoionophoren wurde eine Art Mischung zwischen I und II verwirklicht: Die Lichtabsorption wird bei ihnen dadurch beeinflusst, dass in den Kronenteil des Farbstoffmoleküls selektiv ein Kation eingelagert werden kann, das dann die Lichtabsorption des Komplexes beeinflusst.

Die Komplexierung erfolgt kationenselektiv (im Ionophorteil); die Lichtabsorption im Chromophorbereich z.B. von 1 wird dabei durch das Kation erheblich verändert, wie Fig. 2 beispielhaft zeigt [1].

Auch eine chiroselektive Beeinflussung der Lichtabsorption [2] ist möglich: mit Hilfe des chiralen Chromoionophors 2 gelang Misumi und Mitarbeitern die Unterscheidung chiraler Amine durch unterschiedlich starke (chiroselektive) Farbeffekte [2].

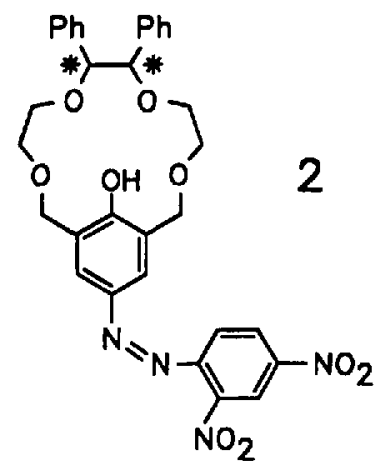

\section{Molekulare Azo-Schalter}

Molekulare Schalter vom Kronen-Typ, die auf einer Azo-Gruppierung beruhen, sind zuerst von Shinkai beschrieben worden (Schema 1) [3]. Die selektive Aufnahme eines Kations im Kronenether-Teil kann durch Licht beeinflusst («geschaltet», modifiziert) werden.

Von besonderem Interesse erscheinen uns Hohlräume, die erst nach Lichteinwirkung $(h v)$ eine zur Komplexierung von Gastmolekülen geeignete Wirtstruktur annehmen, oder bei denen umgekehrt durch die Belichtung $\left(h v^{\prime}\right)$ eine zur Komplexierung von Gästen ungeeignete Hohlraumgestalt erzeugt wird (Schema 2). Hierzu muss

*Korrespondenz: Prof. Dr. F. Vögtle

Institut für Organische Chemie und Biochemie der Universität Bonn

Gerhard-Domagk-Str. I

D-5300 Bonn 1
Fig. 2. Selektiver lonennachweis via kationselektithochrome Verschiebungen bei Zugabe der angegebenen Salze (Kationen) zur Lösung des Chromoionophors in Acetonitril) 
Schema 1. Molekularer Schalter vom Kronen-Typ mit photochemisch schaltbarer Azo-Gruppierung

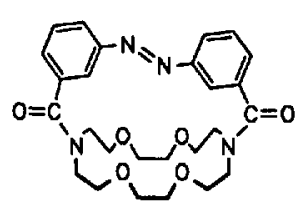

(E) -3

Schema 2. Prinzip eines molekularen Schalters, dessen Hohlraum durch den Schaltvorgang (hv) einen Gast aufnehmen kann, oder einen Gast freisetzt $\left(h v^{\prime}\right)$, der anschliessend als Kupplungskomponente für eine Farbreaktion an der belichteten Stelle dienen kann

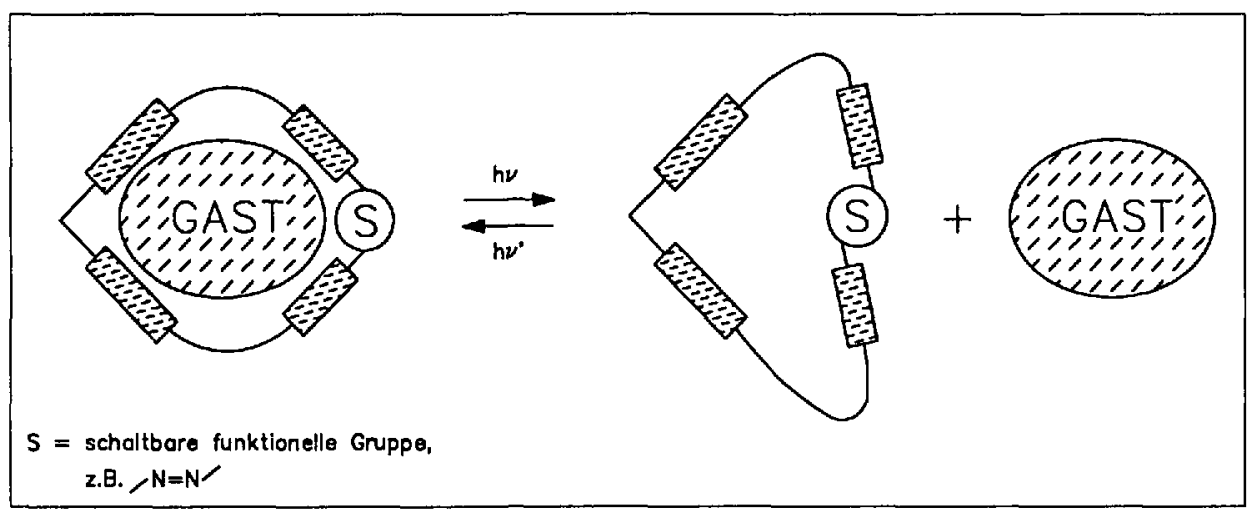

Schema 3

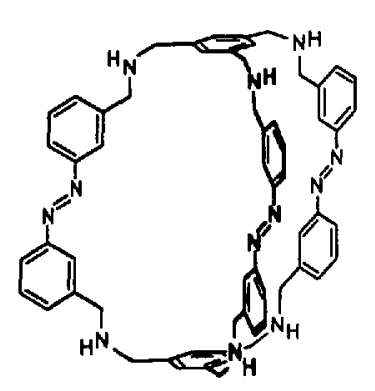

4

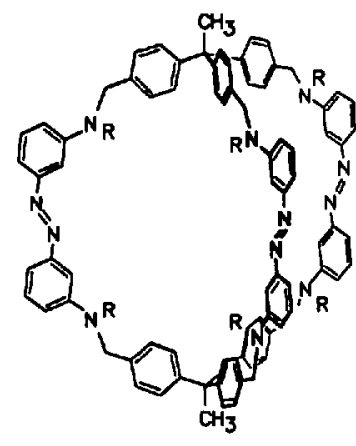

(out/out)

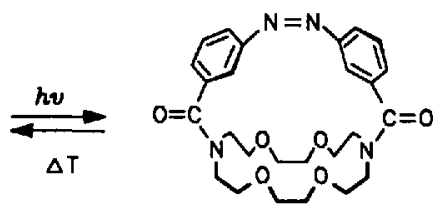

(Z) -3
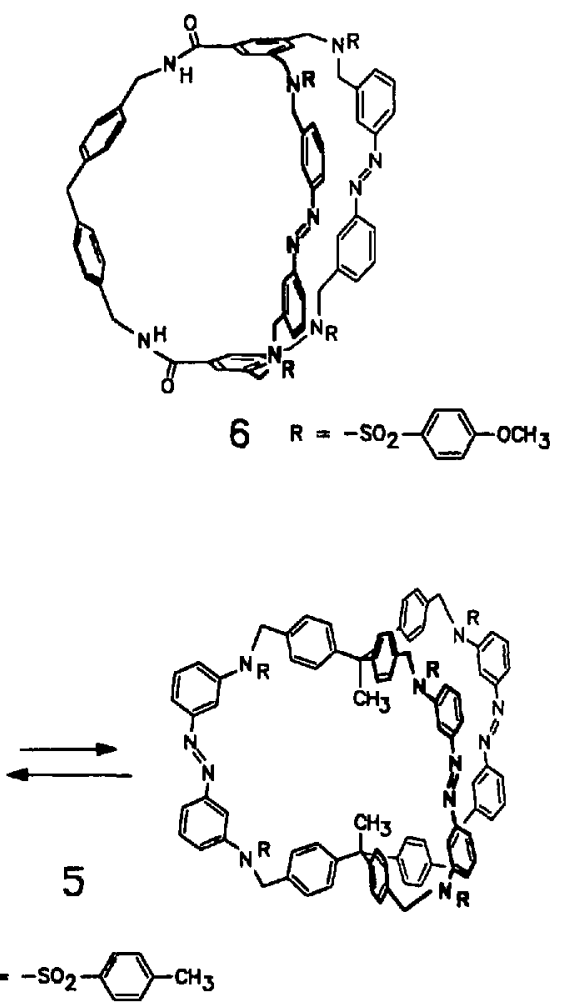

(in/in) grösserem Hohlraum auf TriphenylethanBasis konnte flüssigkeitschromatographisch (HPLC) in die drei zu erwartenden Isomere (outlout, in/in und out/in) aufgetrennt werden. Wie sich zeigen liess, stehen das out/ out- und das in/in-Isomere miteinander im Gleichgewicht.

Bei diesen Untersuchungen stellte sich heraus, dass es besonders wichtig ist, das Azobenzol-System in der meta-Position mit Aminogruppen zu substituieren, da sich dann das photostationäre Gleichgewicht wie gewünscht nur langsam einstellt, so dass die entsprechenden $(E) /(Z)$-Isomere isolierbar und für längere Zeit stabil sind.

Eine weitere von uns angestrebte schaltbare potentielle Wirtstruktur stellt der Makrobicyclus $\mathbf{6}$ dar, der aus einer hydrophoben Diphenylmethan-Einheit und zwei schaltbaren Azo-Gruppierungen aufgebaut ist. Der (gewinkelte) Diphenylmethan-Baustein sollte die Einlagerung aromatischer Gastmoleküle begünstigen.

Ein Beispiel für Makrocyclen zur Komplexierung kleiner neutraler, lipophiler organischer Moleküle, die durch Belichtung in ihrer Struktur reversibel veränderbar sind, zeigt 7 :

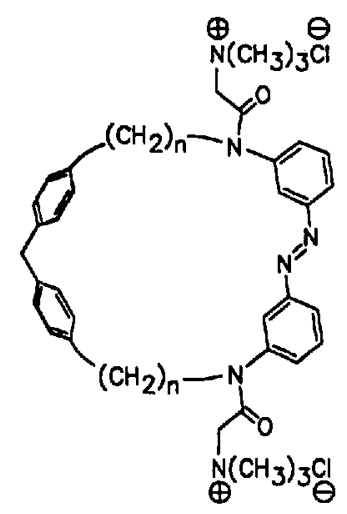

Zur Ausnutzung des «hydrophoben Effekts» als treibende Kraft bei der Komplexierung neutraler organischer Moleküle wurde Wasserlöslichkeit dieses Wirttyps angestrebt. Dies liess sich durch das Anbringen positiver Ladungen in Form quartärer Ammoniumionen erreichen. Das Erzeugen von Ladungen durch Protonierung des Aminoazobenzols muss hierbei vermieden werden, da die thermische Reisomerisierung der Azobenzol-Einheit durch Protonen katalysiert wird [5]. Ausserdem sollten die Ladungen nicht Bestandteil des Makrocyclen-Gerüsts selbst sein, sondern weiter aussen an der Peripherie des Moleküls angebracht werden, um so einen möglichst lipophilen Hohlraum zu gewährleisten.

\section{Fluoreszenzfarbstoffe} chungen hinsichtlich der Lichtabsorption und des Schaltvorgangs führten über die Erzeugung aller vier denkbaren $(E) /(Z)$-Isomere hinaus zu deren analytischer Trennung und Charakterisierung auf der Basis ihrer Lichtabsorption.

Der gleichfalls aus drei Azobenzol-Einheiten aufgebaute Makrobicyclus 5 mit noch
Eine andere Möglichkeit, durch massgeschneiderte supramolekulare Anordnung von $\pi$-Systemen innerhalb eines Moleküls (Typ II) neue Farben, Lichtabsorptionen bzw. Möglichkeiten zur gezielten Änderung von Lichtabsorptionen zu erzeugen, konnten wir pen als Schaltelementen führten zunächst zu dem Grosshohlraum 4 mit drei schaltfähi- 
mit makrocyclisch fixierten Diarylhexatrienen verwirklichen [6]:

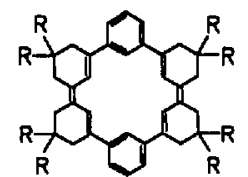

8

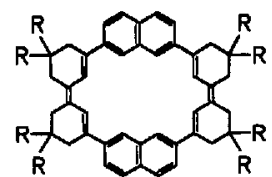

9 a: $\mathbf{R}=\mathrm{H}$

b: $\mathrm{R}=\mathrm{CH}_{3}$

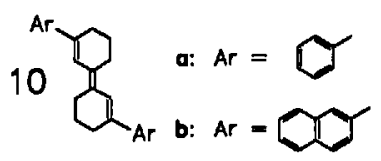

Offenkettige wie auch cyclische Polyene gehören seit vielen Jahren zu den wichtigsten Forschungsgebieten der organischen wie auch der Farbstoff-Chemie [7]. So ist das 1,6-Diphenyl-1,3,5-hexatrien (DPH) z.B. ein vielbenutzter Fluoreszenzindikator, etwa bei Untersuchungen der Molekülanordnung in Vesikeln und Micellmembranen, flüssigkristallinen Phasen und Polymerfolien. Wir versuchen, durch Einbinden bewährter klassischer Chromophor-Bauteile in makrocyclische Systeme neuartige organische Materialien mit besonderen (Lichtabsorptions-) Eigenschaften zu erhalten. 8 und 9 enthalten zwei Diarylhexatrien-Einheiten mit fixierter Geometrie und ohne freie Drehbarkeit der aromatischen Ringe. Im offenkettigen Trien 10a hingegen besteht noch die Möglichkeit der $(E) /(Z)$-Isomerisierung der zentralen exocyclischen Doppelbindung.

Damit ermöglichen die Diarylhexatriene 8-10 die Untersuchung der Auswirkungen von konformativer und konfigurativer Fixierung des jeweiligen Chromophors auf die elektronische Anregung durch UV/VISSpektroskopie (Fig. 3).

Das Fehlen einer - verglichen mit 10a,b - längerwelligen Bande in den Spektren von 8a und 9a bestätigt die Annahme, dass es sich um zwei isolierte $(E, Z, E)$-Diphenylhexatrien-Systeme und nicht um einen einzigen, über den gesamten Cyclus ausgedehnten Chromophor handelt [8]. Auch Untersuchungen an den cyclischen Kationen von 8 und 9 stützen diese Annahme.

Auffällig ist eine gegenüber den Spektren von $10 \mathbf{a}, \mathbf{b}$ zusätzliche Bande $(\lambda=273$ bzw. $286 \mathrm{~nm}$ ), die nach theoretischen Betrachtungen durch das Fehlen eines Symmetriezentrums in 8 und 9 bedingt ist [9], obwohl die nach Rechnungen energetisch günstigste Konformation von 8a ein Inversionszentrum aufweist. Auch dies legt eine Aufteilung der makrocyclischen «Polyen»Cyclen in zwei separate Chromophore nahe.
Kohlenwasserstoff-Ringe des hier beschriebenen Typs bieten sich - besonders bei der Substitution mit acht langkettigen Resten $\mathrm{R}$ - für weitere Manipulationen der Lichtabsorption und -emission an.

Neue konkave Farbstoffmoleküle vom Triphenylmethan-Typ (vgl. 11) zeigen im Gegensatz zu nicht makrocyclischen Vergleichssubstanzen solvatochrome Effekte und reagieren mit bathochromen Farbänderungen auf den Einschluss flacher, aromatischer Gastmoleküle. Sie lassen sich durch $\mathrm{pH}-$ Änderung bezüglich der Farbe reversibel schalten [10]

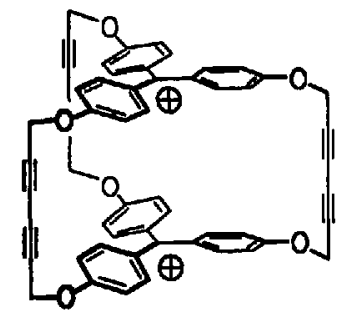

11 (orange)
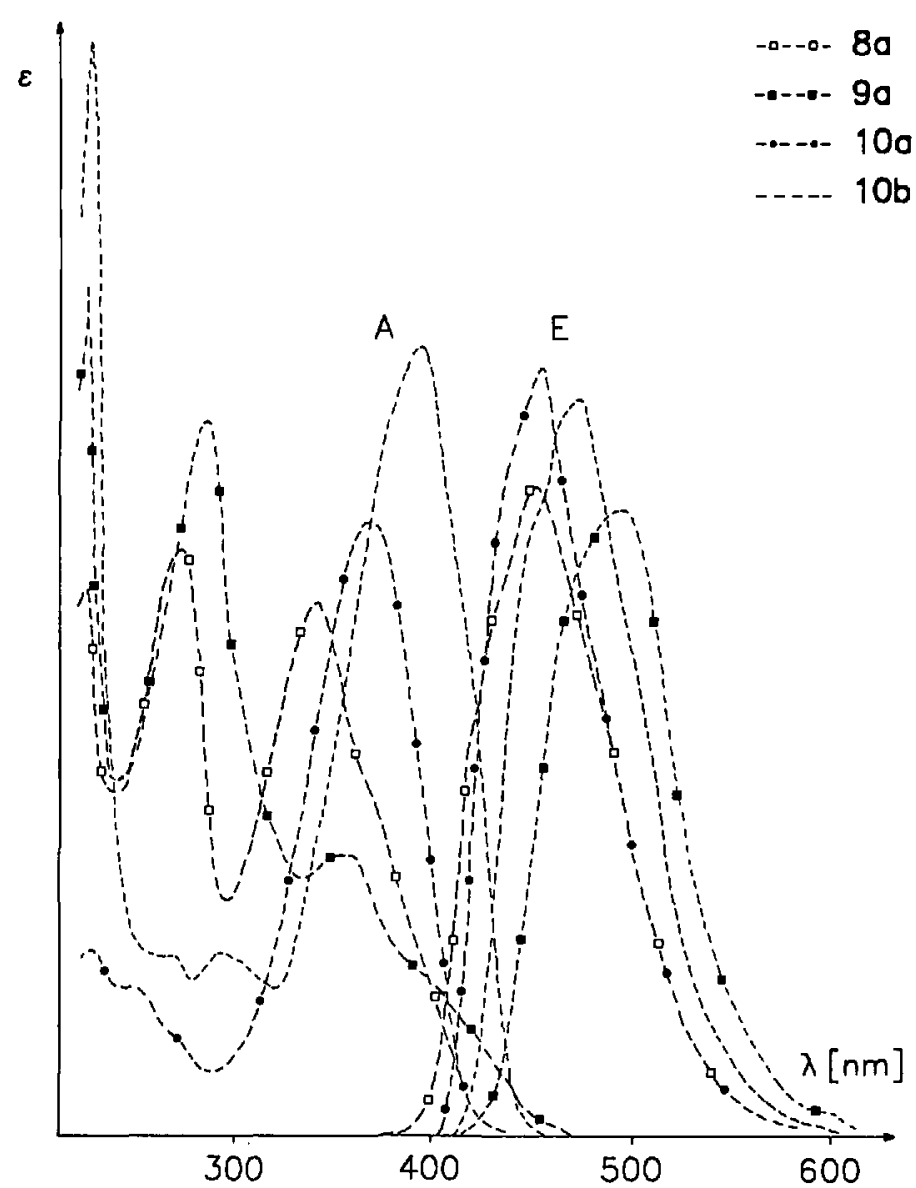

Fig. 3. Absorptions- (A) und Emissionsspektren (E) (in 1,4-Dioxan, T = $292 \mathrm{~K}$, Anregung bei $380-400 \mathrm{~nm}$ ) der Diarylhexatriene $10 \mathrm{a}, \mathrm{b}$ und der Cyclopolyene $8 \mathrm{a}$ und $9 \mathrm{a}, \theta$ in relativen Einheiten
[1] a) J. P. Dix, F. Vögtle, Angew. Chem. 1978, 90 , 893; ibid. Int Ed. 1978, 17, 857; b) J. P. Dix, F. Vögtle, Chem. Ber. 1981, 1/4,638; c) G. Hollmann, F. Vögtle, ibid. 1984, 117,$1355 ;$ d) H.-G. Löhr, F. Vögtle, Acc. Chem. Res. 1985, /8, 65;) C. Reichardt, S. Asharin-Fard, Angew. Chem. 1991, 103, 614; ibid. Int. Ed. 1991, 103, 558.

[2] a) Die enantioselektive Komplexierung mit neutralen chiralen Chromoionophoren wurde bereits 1982 von Vögtle und Mitarbeitern angestrebt: DFG-Antrag Vo 145/34 vom 29.11.1982, vgl. hierzu auch [ld,e]; b) T. Kaneda, K. Hirose, S. Misumi, J Am. Chem Soc $1989,1 / /, 742 ; \mathrm{c}$ ) S. Misumi, Pure Appl. Chem. 1990, 62, 493; d) F. Vögtle, P. Knops, Angew. Chem. 1991, 103 , 972.

[3] a) S. Shinkai, T. Ogawa, T. Nakaji, Y. Kusano O. Manabe, Tetrahedron Lett. 1979, 4569; b) S. Shinkai, T. Nakaji, Y. Nishida, T. Ogawa, O Manabe, J. Am. Chem. Soc. 1980, IO2, 5860.

[4] a) H. W. Losensky, H. Spelthann, A. Ehlen, F Vögtle, J. Bargon, Angew. Chem. 1988, 100, 1225, ibid. Int. Ed. 1988, 27, 1189; b) K. H. Neumann, F. Vögtle, J. Chem. Soc., Chem. Commun. 1988, 520.

[5] G. S. Hartley, J.Chem. Soc. 1938, 633

[6] F. Vögtle, C. Thilgen, Angew. Chem. 1990, 102, 1176, ibid. Int. Ed. 1990, 29, 1162

[7] a) H. A. Staab, 'Einführung in die theoretische organische Chemie', Verlag Chemic, Weinheim, 1964; b) B. M. Krasovitski, B. M. Bolotin, 'Organic Luminescent Materials', VCH, Weinheim, 1988.

[8] a) Vgl. J. Dale, Acta Chem. Scand. 1957, 11,971; b) vgl. auch: H. A. Staab, F. Binnig, Chem. Ber. 1967, 100, 293.

[9] a) L. Zechmeister, A. L. LeRosen, W. A. Schroeder, A. Polgar, L. Pauling, J. Am. Chem. Soc. 1943, 65, 1940; b) R. S. Mulliken. J. Chem. Phys. 1939, 7, 203

[10] R. Berscheid, F. Vögtle, Angew. Chem., zur Veröffentlichung eingereicht. 